\title{
Incluir o replantear: cómo exponer e historizar a las mujeres artistas
}

\author{
Haizea Barcenilla \\ Euskal Herriko Unibertsitatea
}

RESUMEN: El artículo analiza dos exposiciones individuales dedicadas a sendas mujeres artistas de las vanguardias, Hilma af Klint (1862-1944) y Eileen Gray (1878-1976), en museos de reconocido prestigio. Se pretende estudiar la forma en que la articulación del formato expositivo en estos casos trata de legitimar relatos hegemónicos de la historia del arte, intentando integrar en ellos el trabajo de ambas artistas, a pesar de las claras contradicciones que este presenta para dichos relatos. Se presta especial atención a las estrategias discursivas y expositivas utilizadas, haciendo hincapié en la ausencia de lecturas de género, a pesar de las fundamentales aportaciones realizadas desde ese campo sobre ambas artistas.

PALABRAS CLAVE: Exposición, Teoría de género, Hilma af Klint, Eileen Gray, Museos, Lesbianismo, Vanguardias.

\section{Include or Rethink: How to Exhibit and Historize Women Artists}

ABSTRACT: This article analyses two solo exhibitions devoted to women artists from the avantgardes: Hilma af Klint (1862-1944) and Eileen Gray (1878-1976) by prestigious museums. The paper focuses on how the articulation of the exhibition format legitimates hegemonic discourses of art history through integrating both artists' work into them; nevertheless, these women work seems to clearly contradict those discourses. Special attention is paid to the uses of discourse and exhibitionary strategies, in which any interpretation from gender studies is avoided in spite of the fundamental contributions of the field to both women's works.

KEY WORDS: Exhibition, Gender Theory, Hilma af Klint, Eileen Gray, Museums, Lesbianism, AvantGarde.

Recibido: 8 de abril de 2014 / Aceptado: 26 de mayo de 2014.

\begin{abstract}
Lorsqu'on croit parler d'art ou d'oeuvres d'art, bien souvent on ne parle que d'impressions reçues au cours d'une exposition spécifique. Et bien que tout amateur d'art s'en défende, il ne parle jamais d'une oeuvre en tant que telle mais des conditions de sa rencontre avec elle.
\end{abstract} Jérôme Glicenstein ${ }^{1}$

\footnotetext{
* BARCENILLA, Haizea: "Incluir o replantear: cómo exponer e historizar a las mujeres artistas», Boletín de Arte, n. ${ }^{\circ}$ 35, Departamento de Historia del Arte, Universidad de Málaga, 2014, pp. 117-129, ISSN: 0211 8483.

1 "Aunque creamos hablar de arte o de obras de arte, muy a menudo de lo que hablamos es de impresiones recibidas durante una exposición específica. $Y$ a pesar de que todo aficionado al arte lo niegue, nunca habla de una obra de arte como tal, sino de las condiciones de su encuentro con ella". GLICENSTEIN, Jêrome, L'art: une histoire d'expositions, París, Presses Universitaires de France, 2009, p. 10.
} 
Hace años que el formato expositivo es considerado un campo de investigación importante en museología. Desde su diseño hasta su comunicación, la exposición ha sido analizada en múltiples ocasiones puesto que se considera el formato principal de acercamiento al arte y la mejor manera de presentar las colecciones de un museo.

Resulta más sorprendente la relativa poca importancia dada al formato y al discurso expositivo al estudiar la propia historia del arte. Jérôme Glicenstein recuerda que no observamos jamás una obra de arte neutra, incondicionada, libre y sin connotaciones, sino que la conocemos en un contexto construido y mediatizado, que coloca a esa obra dentro de una serie de discursos. La decisión de ubicarla en ese lugar y el relato en el que se enmarca nunca son casuales; es más, dicho contexto de visibilidad puede, a través de su nivel de legitimación, facilitar la inscripción de ciertas ideas y artistas en los engranajes de la historia del arte. No obstante, la importancia de las circunstancias de lo que podríamos llamar el momento de hacer el arte público ha sido generalmente ignorada.

Llama especialmente la atención esta carencia en el estudio del arte contemporáneo, donde la exposición ha jugado a menudo un papel concluyente en la construcción de la historia de determinados movimientos y objetos artísticos². Son escasas las aportaciones fundamentales en este sentido, como la de Staniszewski ${ }^{3}$, en la que analiza los formatos y, relacionada con estos, la ideología latente en la política expositiva del MoMA, o la de Poinsot refiriéndose a las estrategias artísticas para controlar el modo de exposición del trabajo ${ }^{4}$. Otros trabajos, como el de Guasch ${ }^{5}$, proporcionan datos informativos esenciales aportando valiosa información sobre estos eventos, aunque sin analizar en profundidad los discursos que tejen esas formas.

A pesar de este vacío, o tal vez precisamente por él, el poder de la exposición no pasa desapercibido, sobre todo para aquellas que se plantean cómo y

2 Se encuentran estudios interesantes sobre género y espacios expositivos en otras disciplinas y épocas históricas, como el artículo "Exposición y género: el ejemplo de los museos de arqueología" de Isabel Izquierdo Peraile, Clara López Ruiz y Lourdes Prados Torreira, publicado en ASENSIO, Mikel; POL, Elena (eds.), Nuevos Museos, Nuevas Sensibilidades, Series de Investigación lberoamericana de Museología, año 3, vol. 4, pp. 1-181, y SADA CASTILLO, Pilar, "¿Mujeres invisibles? La presencia de la mujer en los discursos expositivos de la Historia», en DOMínGUEZ ARRANZ, Almudena (ed.), Mujeres en la Antigüedad Clásica, género, poder y conflicto, Madrid, Sílex, pp. 229-247.

3 STANISZEWSKI, Mary Ann, The Power of Display. The History of Exhibition Installation at the Museum of Modern Art, Boston, MIT Press, 1998.

4 POINSOT, Jean-Marc, Quand l'oeuvre a lieu. L'art exposé et ses récits autorisés. Les presses du réel, Ginebra, 2008.

5 GUASCH, Anna Maria, El arte del siglo XX en sus exposiciones. 1945-2007, Barcelona, Ediciones del Serbal, 2009. 
desde dónde se ha escrito la historia. Las exposiciones han dado que hablar en el feminismo, y han sido un formato fundamental para experimentar con aproximaciones alternativas y más inclusivas, desde grandes eventos como fue Women Artists 1550-1950, de Linda Nochlin y Ann Sutherland Harris en 1977 en Los Ángeles, hasta entornos más cercanos, como en la programación del Centro Cultural Montehermoso en Vitoria entre 2008 y 2011. Griselda Pollock incluso utiliza la exposición como motor de su Encuentros en el museo feminista virtual, definiéndola como "encuentro que se abre a nuevas relaciones críticas entre las obras de arte, y entre los espectadores y las obras, que apuntan a narrativas reprimidas en las historias del arte ${ }^{6}$.

Mas la exposición aparece para el feminismo antes como formato práctico de acción que como objeto de análisis ${ }^{7}$. Raramente se estudian exposiciones contemporáneas que no se definan como feministas, aunque contribuyan a construcciones históricas que atañen al feminismo. Este artículo ofrece un ejemplo de este tipo de análisis, fijándose en dos importantes figuras recientemente recuperadas por la historia del arte de las vanguardias y en la forma en que han sido expuestas: Hilma af Klint en el Moderna Museet de Estocolmo ${ }^{8}$ y Eileen Gray en el Centro Pompidou de París ${ }^{9}$.

\section{Recuperación, reconocimiento o replanteamiento}

En 2013 han sido abundantes las exposiciones en centros internacionales dedicadas a artistas mujeres de las vanguardias que, por diferentes razones, no habían merecido antes tanta atención ${ }^{10}$. Es el caso de Hilma af Klint, cuya obra abstracta no fue conocida durante su vida por el miedo que le producía la posible reacción pública; esta inseguridad Ilevó a la artista a estipular en su testamento

6 POLLOCK, Griselda, Encuentros en el museo feminista virtual, Madrid, Cátedra, 2010, p. 61.

7 En caso de análisis, a menudo el objeto de estudio son exposiciones realizadas desde el feminismo, como en FERNÁNDEZ LÓPEZ, Olga, "Feminismos en los discursos expositivos y relatos museográficos en España desde los años noventa», en Genealogías feministas en el arte español: 1960-2010, comisariada por Patricia Mayayo, autora esta última también del artículo "Después de Genealogías Feministas. Estrategias feministas de intervención en los museos y tareas pendientes" en Investigaciones feministas, Madrid, UCM, vol. 4, pp. 25-37.

8 Hilma af Klint-A Pioneer in Abstraction, Moderna Museet de Estocolmo. 16 de febrero-26 de mayo 2013. La misma exposición itineró posteriormente en el Hamburger Banhof de Berlín y en el Museo Picasso de Málaga.

9 Eileen Gray, Centre Pompidou de París. 20 de febrero-20 de mayo 2013. La misma exposición itineró posteriormente al Irish Museum of Modern Art.

10 Es el caso de María Blanchard en el Reina Sofía o de la pintora abstracta libanesa Saloua Raouda Choucair en la Tate Modern de Londres. 
la ocultación de sus trabajos hasta veinte años tras su muerte. Sus pinturas se dieron a conocer por primera vez en $1986^{11}$ y la exposición en el Moderna Museet es el fruto de varios años de investigación.

Eileen Gray, en cambio, sí gozó de momentos de reconocimiento en vida. No obstante, diferentes factores entre los que se han mencionado la tensa relación que mantenía con Le Corbusier y el posible rechazo que el arquitecto sentía por las ideas subyacentes en su trabajo ${ }^{12}$, junto con su reconocida timidez ${ }^{13}$ y la erradicación de su nombre en artículos sobre sus obras a partir de los años $30^{14}$, propiciaron la desaparición de Gray de la escena pública en los años 30, que solo comenzó a invertirse a partir de los años 70, poco antes de su muerte ${ }^{15}$.

Por tanto, nos encontramos ante dos artistas que no fueron contempladas en el momento de construcción de los cánones de la historia del arte de vanguardias, a las que se dedica ahora por primera vez una exposición retrospectiva con trabajos fundamentales ${ }^{16}$. El hecho de recibir esta atención por parte de museos nacionales consagrados significa un reconocimiento y un intento de incluirlas en las clasificaciones expuestas por esos museos, referencias fundamentales a la hora de escribir la historia del arte.

Pero desde un prisma de análisis de género, la simple inclusión de elementos puntuales en las reglas admitidas de la historia del arte puede no ser suficiente. Hace ya cuarenta años que Linda Nochlin puso sobre la mesa que el arte es una construcción cultural influida por complejos factores sociales, en contra de la difundida idea de que la capacidad creadora es un don natural e intrínseco con el que los artistas nacen. Así, cuando revisamos e incluimos a artistas mujeres sin analizar las razones por las que su trabajo no fue apreciado en su época o pasó al olvido en décadas posteriores, y sin poner en cuestión los valores que han establecido cánones excluyentes e inalterables, estamos aceptando una gran narrativa de la historia del arte, basada en la idea del genio, de la autonomía y de círculos cerrados a los que, simplemente, añadimos personajes

11 Una selección de sus obras se mostró en The spiritual in Art: Abstraction Painting 1890-1985, Los Angeles County Museum of Art, comisariada por Maurice Tuchman.

12 RAULT, Jasmine, "Occupying E.1027. Reconsidering Le Corbusier's "gift" to Eileen Gray», Space and Culture, SAGE Journals, vol. 8, mayo 2005, pp. 160-179.

13 ADAM, Peter, Eileen Gray: Architect, Designer, a Biograph, Nueva York, Harry N. Abram, 2000, p. 257. 14 COLOMINA, Beatriz, Doble exposición, Madrid, Akal, 2006, pp. 62-69.

15 RAULT, Jasmine, en Eileen Gray: new angles in gender and sexuality, tesis doctoral en McGill University, Montreal. Library and Archives Canada, Ottawa, 2006, pp. 149-186, analiza la posibilidad de que el retiro de Gray estuviera unido con la campaña pública contra el lesbianismo llevada a cabo en 1928 con el juicio por obscenidad a Radclyffe Hall y las consecuencias sociales de la mediatización de la imagen de las lesbianas, acompañada del correspondiente rechazo.

16 En el caso de Eileen Gray, había sido objeto de otras exposiciones, pero no conjuntamente lo que se considera la época déco y la modernista, por lo que se considera la primera retrospectiva completa. 
secundarios. Esta es también la tesis de la anteriormente citada Griselda Pollock, y la vigencia de sus planteamientos se hace evidente con la traducción al español en 2013 de su libro de 1988 Visión y diferencia. Feminismo, feminidad e historia del arte, que sigue siendo una referencia fundamental ${ }^{17}$.

Por ello, aunque sea positivo empezar a incluir protagonistas diferentes en los relatos de las vanguardias, no debemos dejarnos llevar por el conformismo. Precisamente porque organizar exposiciones hoy, en un momento en el que acceder al conocimiento y a investigaciones paralelas es mucho más sencillo, y cuando el público de los museos nacionales se ha masificado, es una gran responsabilidad pública.

\section{La primacía del genio}

Aunque se suponga que el genio es único e incomparable, la Historia del Arte sigue basándose en ideas de superioridad y éxito. Aún hoy, se dedica gran esfuerzo a dictaminar quién utilizó antes cierta técnica o abordó cierto concepto, dejando en segundo nivel las conversaciones, colaboraciones y procesos que condujeron a dichos resultados. Esto también se refleja en la tendencia a medir quién tuvo más discípulos sin preguntarse cuáles fueron las razones de una mayor o menor visibilidad de ciertas obras, que facilitaría su conocimiento e influencia sobre otros. La idea de competición no se aleja del mercado del arte, donde se transmite de forma engañosa que el buen arte es el que más cuesta, uniendo perversamente la supuesta calidad objetiva y la economía.

El título de la exposición del Moderna Museet, Hilma af Klint, pionera de la abstracción, recibe al visitante con esta lógica. No resulta sorprendente en un museo cuya anterior exposición se titulaba Picasso versus Duchamp. He was wrong y se basaba en establecer cuál de los dos artistas fue más influyente en el siglo XX. La frase elegida como subtítulo ("estaba equivocado») se atribuye a Picasso, refiriéndose a Duchamp cuando tuvo noticia de su muerte, y presenta a los dos artistas como genios eternamente contrapuestos.

Hilma af Klint nació en 1862 en la capital escandinava y fue una de las primeras mujeres que, además de seguir una formación artística en la Real Escuela de Artes de Estocolmo, se dedicó profesionalmente a la pintura. Desde su graduación en 1887 hasta 1908 trabajó en los céntricos estudios que brindaba la

17 POLLOCK, Griselda, Visión y diferencia. Feminismo, feminidad e historia del arte, Buenos Aires, Fiordo, 2013. 
escuela a graduados con talento. A pesar de que en vida principalmente vendía y exponía paisaje naturalista, lo que se visitaba en el Moderna Museet era una parte de su obra muy diferente, ingente en tamaño y cantidad: grandes composiciones que, oscilando entre la figuración y la abstracción, intentaban expresar el origen del mundo. Estas composiciones permanecieron ocultas durante su vida, y no se dieron a conocer hasta los años $80^{18}$.

La razón de ser de este trabajo, escondido hasta finales del siglo XX, está íntimamente relacionada con el profundo interés por comprender aquello que queda fuera del campo de lo visible, de lo físico. Desde finales del siglo XIX, una fuerte tendencia hacia el ocultismo se había expandido por Europa y EE. UU. Descubrimientos científicos como los rayos $X$ en 1895, o las ondas electromagnéticas, incentivaron la certeza de que existen fuerzas más allá de lo visible ${ }^{19}$. La teosofía, doctrina de búsqueda espiritual fundada en 1875, era estudiada con atención por artistas como Kandinsky o Mondrian ${ }^{20}$, que intentaban representar en su arte las fuerzas espirituales inherentes al alma.

En el caso de Hilma af Klint, su interés por lo oculto se articuló a través del espiritismo, que practicó desde muy joven. En 1896 formó un grupo Ilamado De Fem (Las Cinco) con Anna Cassel, también artista y compañera de estudios, Sigrid Hedman, Cornelia Cederberg y Mathilde N. Durante diez años Ilevaron a cabo sesiones de espiritismo, en las que recibían mensajes de otra dimensión, que transcribían en textos y dibujos automáticos. En esas sesiones Hilma af Klint recibió lo que denominó "el encargo" de sus Pinturas para el Templo por parte de un espíritu Ilamado Amaliel ${ }^{21}$, consistentes en varias series que intentaban explicar los ciclos de la vida. El origen de estas creaciones, extraordinariamente modernas y lejanas del mundo consciente, hicieron dudar a af Klint sobre la capacidad del público para comprenderlas.

La dedicación de estas cinco mujeres al espiritismo se comprende dentro de un contexto más amplio. Desde mediados del siglo XIX, el movimiento de emancipación de la mujer mantuvo una estrecha relación con el espiritismo, como ha demostrado Ann Braude en el caso de Estados Unidos. No es casual que la mayoría de médiums conocidas, en Europa como en América, fueran mujeres. Como señala Braude, las características consideradas puramente fe-

18 MULLER-WESTERMANN, Iris (com.), Hilma af Klint. A pioneer of abstraction, Estocolmo, Moderna Museet, 2013, p. 33 (edición española en Málaga, Museo Picasso Málaga, 2013).

19 Ibid., p. 41.

20 RINGBOM, Sixten "Art and the Epoch of the Great Spiritual: occult elements in the early theory of abstract painting", Journal of the Warburg and Courtauld Institutes, 29, 1966, pp. 386-418.

21 MULLER-WESTERMANN, Hilma af Klint..., p. 41. 
meninas (constitución delicada y capacidad de excitación nerviosa) resultaron apropiadas para el contacto con los espíritus; y así, las mujeres encontraron un espacio en el que su individualidad era aceptada como igual o incluso superior a la de los hombres. El espiritismo implicaba que, por primera vez, se escuchaba y respetaba la voz de la mujer, precisamente porque dicha voz no se consideraba propia. Así, el espiritismo permitió a muchas mujeres autonomía y capacidad de liderazgo ${ }^{22}$. Un claro ejemplo de ello sería la propia Madame Blavatsky, fundadora de la teosofía.

Esta información contextual, fundamental para la lectura crítica del trabajo, se encuentra ausente en la exposición. Los textos de sala mencionan la importancia de los descubrimientos científicos y del interés por lo oculto, sin llegar a una lectura contextual relacionada con el género de la artista y su actividad de médium, de manera que la importancia política de esta se diluye. No solo eso: en su intento por hacerla figurar entre una serie de genios individualizados y autónomos, af Klint se presenta como una visionaria solitaria que trabajaba escondida en su estudio. Incluso el capítulo que la comisaria Iris Müller-Westermann le dedica en el catálogo se titula "Hilma af Klint: una pionera de la abstracción en aislamiento", a pesar de relatar cómo el estudio de Hamngatan era compartido con otras dos artistas, que durante diez años realizó sesiones semanales con $D e$ Fem, y que el ambiente de af Klint se centraba en comunidades de mujeres, y era por lo tanto paralelo al del arte oficial de Estocolmo ${ }^{23}$.

La obra se presenta como si de una experiencia puramente estética se tratara, de una pelea por el genio mayor con pintores como Kandinsky o Malevich. En vez de aprovechar la oportunidad para cuestionar la lógica competitiva de genios solitarios, y analizar en cambio círculos artísticos formados por artistas y pensadoras de la época, se sigue una dinámica de aceptación acrítica de las narrativas ya establecidas, a pesar de que el trabajo de af Klint venga precisamente a contradecirlas. Esta estrategia no contempla que, al someterse a esos cánones de genialidad sobresaliente, difícilmente se va a aceptar a una nueva maestra que debería haber sobresalido en vida. Y si se llegara a aceptar, como ocurre con Mary Cassat, será como excepción que confirma la regla: una regla inamovible en la que las mujeres siempre necesitan hablar con la voz de otro.

22 BRAUDE, Ann, Spiritualism and women's rights in Nineteenth Century America, Bloomington, Indiana University Press, 2001.

23 MULLER-WESTERMANN, Hilma af Klint..., pp. 33-48. 


\section{¿Lo personal es artístico?}

A la exposición de Eileen Gray sí debe reconocérsele un esfuerzo crítico, en su caso, en lo referente a la división entre artes mayores y menores: se exponen al mismo nivel todos los trabajos que Gray realizó, desde diseños de alfombras hasta edificios. Un avance importante, puesto que es necesario revisar la división entre disciplinas cuando nos referimos al arte de vanguardias: una de las grandes aportaciones de la época fue el acercamiento entre las Ilamadas Bellas Artes y las artes utilitarias, antecedente fundamental del arte contemporáneo. Precisamente Eileen Gray (al igual que otras artistas, como Sonia Delaunay), muestra una trepidante capacidad de romper con dichas barreras y de integrar el arte y la vida en un solo campo de acción, más amplio y conceptual.

Eileen Gray (1878-1976), de origen irlandés y familia adinerada, se formó en la Slade School of Art de Londres. Gracias a su posición pudo viajar y vivió la mayor parte de su vida en París. Sus primeros trabajos, además de dibujos, fueron muebles y utensilios en laca, una técnica de China y Japón que dominó, así como una línea de textiles realizados en colaboración con Evelyn Wyld. A partir de 1920 incluye otros materiales en su diseño de interiores, presentados en la tienda de decoración y diseño Jean Désert que funda en 1922. Entre 1926 y 1929 construye E1027, una casa que se convertirá tardíamente en un ejemplo fundamental para la arquitectura moderna. Le siguen otras dos construcciones y una serie de proyectos que no llegaron a realizarse, todos ellos representados en la exposición. Una muestra, por lo tanto, que no discrimina en función de disciplinas artísticas, y comprende el trabajo de Gray como una unidad conceptual. A pesar de todo, hay un gran ausente en la muestra: el entorno personal de la artista, una ausencia aún más llamativa por la citada importancia de la ruptura en la división entre el arte y la vida.

En efecto, uno de los caballos de batalla del feminismo ha sido la cuestión del papel de la vida personal de las artistas en el análisis de su obra. La biografía, desde Vasari basada en anécdotas que demostraban la genialidad del homenajeado, ha sido criticada por la historiografía del arte, que ha intentado construirse como una ciencia objetiva. Así, las circunstancias personales y sociales, elementos como el género o la clase social, han sido considerados factores de segundo nivel y, paradójicamente, lo personal sólo se ha conservado en el relato en caso de reiterar el aura de genio del artista estudiado. Esta visión ha sido deconstruida desde la investigación feminista, como hace la propia Pollock al contestar el trabajo de T. J. Clark sobre el impresionismo, subrayando la imposibilidad de las 
mujeres impresionistas para experimentar el mismo concepto de modernidad que sus compañeros masculinos, puesto que era imposible para ellas acceder a los lugares que configuraban el estilo de vida moderno masculino, como bares, cabarets y prostíbulos ${ }^{24}$. El estudio de las mujeres artistas, sin caer en sentimentalismos y anécdotas, no puede ignorar que el hecho social de su género ha condicionado absolutamente el trabajo artístico. Esto se acentúa si además de mujer el sujeto de estudio es lesbiana, como probablemente es el caso de Eileen Gray.

De hecho, en los últimos años las más relevantes contribuciones al estudio de Gray se han dado desde perspectivas de género y estudios del lesbianismo. Aportaciones como las de Colomina ${ }^{25}$, Walker ${ }^{26}$ o Rault ${ }^{27}$ son fundamentales. Es difícil definir la sexualidad de Gray -los estudios mencionan que la imagen de la identidad "lesbiana» no empezó a perfilarse hasta 1928, por lo que Jasmine Rault ${ }^{28}$ opta por denominarla «no-heterosexual»-, pero se puede asegurar que mantuvo relaciones íntimas con varias mujeres y, en un caso conocido, con un hombre ${ }^{29}$. Su círculo de amistades, desde su llegada a París, se concentró en torno a su vivienda en la Rive Gauche, en una zona en la que vivían importantes escritoras y artistas lesbianas como Natalie Barney, Gertrude Stein o Romaine Brooks. Mantuvo una estrecha relación con Gabrielle Bloch, compañera sentimental de Loïe Fuller, quien era además la encargada de la contabilidad de su estudio de diseño, y muchas de las mujeres de este círculo fueron sus clientas ${ }^{30}$.

Dando relevancia a estas características y relaciones personales, el enfoque de la investigación de Jasmine Rault varía de las anteriores llevadas a cabo por Adam $^{31}$ y Constant ${ }^{32}$. Estos trabajos fueron fundamentales para incluir a Gray como personaje clave del modernismo, y le atribuían la característica de añadir toques de sensualidad en lo que Constant denominó un «modernismo no-heroico». En ambos casos se compara a Gray con sus contemporáneos masculinos

24 POLLOCK, Griselda, Vision and Difference: Feminity, Feminism and Histories of Art, Londres, Routledge, 1988, pp. 50-90.

25 COLOMINA, Beatriz, "War on Architecture", Assemblage, vol. 20, The MIT Press, 1993, pp. 28-29; "Battle Lines: E.1027» en HUGHES, Francesca (ed.), The Architect: Reconstructing Her Practic, Cambridge, The MIT Press, 1996, pp. 2-25; Doble exposición...

26 WALKER, Lynn, "Architecture and Reputation: Eileen Gray, Gender, and Modemism» en SPARKE, Penny \& MARTIN, Brenda (eds.), Women's Places: Architecture and Design 1860-1960, Routledge, Londres y Nueva York, 2003.

27 RAULT, Eileen Gray..., p. 71.

28 Ibid., p. 7.

29 Gray quemó todas sus cartas personales antes de su muerte, por lo que las relaciones que la unían a muchas personas no están completamente confirmadas.

30 Ibid., p. 71.

31 ADAM, Eileen Gray...

32 CONSTANT, Caroline, "E.1027: The Nonheroic Modernism of Eileen Gray", Journal of the Society of Architectural Historians, vol. 25, septiembre 1994, pp. 265-279. 
(Le Corbusier, Badovici, Oud). Rault, por el contrario, propone analizar a Gray en su propio contexto de intercambio y trabajo, relacionando su obra con la de Romaine Brooks, Radclyffe Hall y Djuna Barnes. Estas uniones muestran mayor afinidad que la que se intenta establecer con el modernismo oficial, y llevan a Rault a defender la existencia de una estética moderna sáfica (siguiendo la definición acuñada por Shari Benstock ${ }^{33}$ ), elaborada por la comunidad de artistas no heterosexuales a la que Gray era próxima, y a la que el propio trabajo de Gray responde, como creadora de espacios de vida y encuentro para esta comunidad y para ella misma ${ }^{34}$.

A pesar de la lógica de la aportación de Rault, en la reciente exposición todo punto de vista de género es obviado por la comisaria Cloé Pitiot, ocultando el entorno que rodeaba a Gray y su sexualidad. Los trabajos de Colomina son despachados en el catálogo en una nota al pie por considerarse que prestan más atención a las actuaciones de Le Corbusier sobre E1027 que al propio trabajo de Gray $^{35}$. En cambio, la timidez de la artista, a la que se atribuye su retiro y vida privada, sí se considera relevante. La investigación de Rault aparece en la bibliografía pero es ignorada en la sala de exposiciones y en el catálogo. Así, las relaciones personales de Gray son mencionadas con una supuesta objetividad y distancia que, en realidad, oscurecen las condiciones sociales de producción de su obra y la colocan en un espacio indefinido en el que la artista es un ente sin sentimientos ni experiencias personales. Aunque colaboró durante periodos prolongados y fundamentales en su trabajo con otras mujeres no heterosexuales (como Gabrielle Bloch y Evelyn Wyld), y Natalie Barney la incluyó como amiga en su ilustración para Aventures de l'esprit ${ }^{36}$, estas mujeres no merecen un espacio relevante en sala. Más aún, en la sección en la que se exponen fotografías de sus clientes, nos encontramos con la propia Barney, Romaine Brooks, Gertrude Stein, Elizabeth Eyre de Lanux, Isadora Duncan y Loïe Fuller. En el texto que las acompaña se habla de manera extensa de Jacques Doucet, la venta de cuya colección sacó a la luz en 1972 el trabajo de Gray, y en las últimas tres líneas se menciona someramente que «desde 1922 a 1930 la clientela de Gray incluía a

33 BENSTOCK, Shari, "Expatriate Sapphic Modernism: Entering Literary History," en JAY, Karla \& YOUNG, Allen, Lesbian Texts and Contexts: Radical Revisions, Nueva York, New York University Press, 1990, p. 183; Women of the Left Bank: Paris, 1900-1940, Austin, University of Texas Press, 1986. Citados en RAULT, Eileen Gray..., p. 192.

34 RAULT, Eileen Gray..., pp. 186-193.

35 MIGAYROU, Frédéric, "Esthétique d’Eileen Gray: dissynchronies de la mesure», en PITIOT, Cloé (com.), Eileen Gray (MNAM), París, Centre Georges Pompidou, 2013, p. 216.

36 RAULT, Eileen Gray..., p. 19. 
aristócratas, diseñadores de moda, financieros, mujeres escritoras y artistas. ${ }^{37}$ Se evita mencionar que más de la mitad de las personas representadas pertenecían a ambientes lésbicos, eludiendo conscientemente profundizar en ninguna de las relaciones personales en torno a Gray. El estudio de Rault muestra similitudes entre el trabajo de Brooks, Hall y Barnes que contribuyen de manera importante a la comprensión de sus diseños, pero que se excluyen premeditadamente de la exposición y su catálogo ${ }^{38}$.

La vida y amistades femeninas de af Klint también brillan por su ausencia. A pesar de que Müller-Westermann ${ }^{39}$ afirma que su entorno estaba compuesto por mujeres, en ningún momento se les dedica la más mínima investigación, por lo que quedan en la indefinición. En la exposición no se menciona que af Klint no se casó, y que pasó veinte años viviendo con Thomasine Andersson, también antropósofa y antigua enfermera de su madre. Andersson solo se menciona muy superficialmente en la cronología del catálogo, sin explicar la relación que pudiera haber entre ellas. En la exposición tampoco se menciona el nombre de sus cuatro compañeras en De Fem durante veinte años, y sus colegas de estudio no llegan a nombrarse ni tan siquiera en el catálogo. Sí hay esfuerzos por dilucidar el contacto que af Klint pudo establecer con hombres distinguidos, como Rudolf Steiner, y se especula sobre la posibilidad de que pudiera visitar una exposición de Edvard Munch en Estocolmo. Se obvian posibles relaciones estilísticas con la también artista Anna Cassel, de De Fem, y se buscan las que pudieran existir con Kandinsky o Kupka, cuyo trabajo seguramente nunca vio. Tanto en la exposición como en el catálogo es evidente el deseo de unirla a una historia del arte ya consagrada, sin explorar el contexto en el que realmente se movía, y que pudo producir otras obras interesantes, hoy igualmente desconocidas.

Esta persistencia en obviar los contextos personales de las artistas se debe a que la historia del arte canónica, como ya hemos mencionado, rechaza la biografía dentro de su suposición de objetividad. Esta regla puede aplicarse con muchísima más facilidad a aquellos que no eran segregados por su sexo, sexualidad o color de piel, y que tenían libertad de elegir entre diferentes estilos de vida; que no necesitaban contraer matrimonio para que alguien los mantuviera, un factor que también resultó decisivo para que Gray y af Klint pudieran dedicarse al arte, ya que contaban con patrimonio propio. En caso contrario,

37 Texto de cartela en sala.

38 Considero que el hecho de que la referencia a Rault aparezca en la bibliografía debe significar que aunque Pitiot, la comisaria, conocía su trabajo, ha decidido no tenerlo en cuenta.

39 MULLER-WESTERMANN, Hilma af Klint..., p. 38. 
probablemente se habrían visto abocadas a casarse y dedicarse al cuidado de su familia, o habrían tenido que llevar a cabo trabajos que habrían imposibilitado su actividad artística. Es evidente que la neutralidad y objetividad de la historia del arte sólo puede construirse sobre un supuesto vacío de condiciones sociales que en la vida real tenían un efecto directo sobre la visibilidad y legitimación del trabajo de las artistas.

Otra consecuencia de la lógica del genio es la falta de atención al trabajo colaborativo, evidente sobre todo en la exposición de af Klint. El trabajo que realizó con De Fem queda ensombrecido en sala: se le dedica un pequeño espacio al final de la exposición, sin mencionar los nombres de las otras cuatro mujeres ni la estrecha relación que las unió durante veinte años. Los textos que escribían mediante escritura automática se presentan sin traducción del sueco, al contrario que todo el resto de información. De Fem fue sin duda un importante grupo de apoyo para af Klint, pero su relación continuada contradice la lectura de genio solitario que la exposición pretende imponerle.

El trabajo de Eileen Gray también se llevó a cabo eminentemente en colaboración. Para la laca, con el japonés Seizo Sugawara; para los textiles, con la tejedora Evelyn Wyld. La casa E-1027 la realizó con el arquitecto Jean Badovici, y así sucesivamente. En la exposición, los objetos están atribuidos correctamente, y se habla de sus relaciones profesionales con Sugawara y Badovici; no obstante, la importancia de la colaboración como parte fundamental de su forma de trabajo no acaba de encontrar forma expositiva. El propio formato, pensado para resaltar la autoría y la cronología ${ }^{40}$, hace que la información sobre las colaboraciones resulte confusa: en vez de mostrar relaciones a largo plazo (como fueron las establecidas con Sugawara y Wyld) se crea una contradicción entre la predominancia de Gray como autora y la de sus colaboradores como elementos semi-externos cuya labor no se esclarece. Este sistema de catalogación y presentación por individualidades provoca que la expresión de las prácticas colaborativas, comunes entre mujeres, sean muy difíciles de expresar y legitimar, puesto que diluyen la autoría y, con ello, la posibilidad de insertarse en el ranking de genialidad que define tanto la historia del arte como su mercado.

Podemos concluir que, a pesar del avance que supone la exposición de artistas que no recibieron el debido reconocimiento en su época o en épocas inmediatamente posteriores, se debe ser muy crítico con las decisiones curatoria-

40 Las cartelas establecen a través del formato el tipo de información que se transmite: autor, fecha, material. Resulta difícil visibilizar así procesos de trabajo más duraderos, colaboraciones continuadas o autorías múltiples. 
les y museológicas, pues ejercen una influencia directa tanto sobre la visión que se fomenta sobre el arte, como sobre la historización de mujeres artistas. Estas decisiones conllevan que el imaginario colectivo siga basándose en el misticismo de la genialidad, por lo que el propio sistema dificulta la inserción de figuras menos conocidas o contestatarias frente a ese misticismo. Las condiciones de producción que favorecían el trabajo de algunos artistas y su recepción (occidentales, hombres y blancos en su mayoría) son obviadas, cuando en realidad resultaron fundamentales para la realización y el juicio de las obras. Al no preguntarse por las razones que Ilevaron a af Klint a esconder su trabajo mientras Rudolf Steiner o Kandinsky lo mostraban, o por las que el modernismo de Gray es tan diferente al de Le Corbusier y causó tanta incomodidad, se demuestra que no se está escribiendo la historia a partir de las obras de arte y sus aportaciones, asumiendo la posible necesidad de replantear los sistemas y categorías existentes; al contrario, se intentan encajar en una historia ya redactada en la que, por sus propias características, difícilmente tendrán cabida y si la tienen es a costa de perder su singularidad discursiva. Hasta que la propia práctica museográfica y curatorial no replantee de manera crítica sus principios a la hora de mostrar a estas mujeres y sus contextos, será difícil construir alternativas a la forma de comprender la historia del arte, y la valiosa aportación de estas artistas se verá reducida al nivel de anécdota histórica. 
